\title{
Training project as an autonomous learning strategy using a virtual platform for the development of competences
}

\section{Training project as an autonomous learning strategy using a virtual platform for the development of competences}

\author{
DOI: $10.46814 /$ lajdv3n6-016
}

Recebimento dos originais: 01/10/2021

Aceitação para publicação: 26/11/2021

\author{
Dr. René Rodríguez Zamora \\ Escuela de Psicología Mazatlán, \\ Universidad Autónoma de Sinaloa, México \\ E-mail: rene.rodriguez@info.uas.edu.mx \\ Dra. Leonor Antonia Espinoza Núñez \\ Tecnológico Nacional de México Campus Mazatlán, \\ E-mail: leonor.en@mazatlan.tecnm.mx
}

\begin{abstract}
RESUMEN
En esta propuesta se presenta el uso de las nuevas tecnologías como una herramienta de apoyo educativo y como una práctica generada a partir del acercamiento cognitivo y afectivo del estudiante con los contenidos de la asignatura, así mismo tiene la doble intención de iniciar un conjunto de prácticas educativas, mediante la estrategia de proyecto formativo, sobre la base del aprendizaje autónomo, colaborativo y orientado en la investigación, lo que le permite conocer el fenómeno psicosocial estudiado en sus diversos contextos de significación. Esta propuesta se circunscribe en un modelo constructivista que ubica al sujeto que aprende como el principal actor que llega a controlar su propio proceso mediante el establecimiento de un plan de acción que consensa de manera colectiva. Por otra parte se ubica dentro del enfoque por competencias que define el marco de la reforma educativa, contenida en el enfoque por competencias, estableciendo las áreas a desarrollar. En este sentido, se desarrollan las competencias integrando el saber, el saber hacer y el saber ser. Se pretende con esta estrategia atender el desarrollo de competencias mediante la identificación de problemas y la aplicación del proceso investigativo, a través de la metodología de proyectos desarrollando aptitudes y actitudes para el trabajo colaborativo y la incorporación de la tecnologías para un mejor desenvolvimiento de la práctica educativa.
\end{abstract}

Palabras Clave: Proyecto Formativo, Uso de Tecnología, Plataforma Virtual, Desarrollo de Competencias.

\section{BSTRACT}

This proposal presents the use of new technologies as an educational support tool and as a practice generated from the cognitive and affective approach of the student with the contents of the subject, likewise it has the double intention of initiating a set of educational practices, through the formative project strategy, on the basis of autonomous, collaborative and research-oriented learning, which allows him to know the psychosocial phenomenon studied in its various contexts of significance. This proposal is circumscribed in a constructivist model that places the learning subject as the main actor who gets to control his own process through the establishment of an action plan that consents collectively. On the other hand, it is located within the competency-based approach that defines the 
framework of the educational reform, contained in the competency-based approach, establishing the areas to be developed. In this sense, competencies are developed by integrating knowledge, know-how and know-how to be. This strategy is intended to address the development of competencies through the identification of problems and the application of the research process, through the project methodology, developing skills and attitudes for collaborative work and the incorporation of technologies for a better development of the educational practice.

Keywords: Training Project, Use of Technology, Virtual Platform, Competency Development.

\section{INTRODUCCIÓN}

En la presente propuesta, se presenta la importancia de generar estrategias innovadoras que permitan la movilización de los saberes integrando el saber en las tres dimensiones, el saber, el saber hacer y en el saber ser como partes del proceso de aprender. Se basa en el enfoque en competencias a partir de la generación de experiencias de aprendizaje, que sea significativo para el estudiante. En un segundo momento se incluye el papel que juega el uso de la tecnología y sus recursos como medios para generar ambientes de aprendizaje colaborativo además de autónomo con el uso de plataforma virtual en su modelo b-learning como medio de apoyo didáctico en la promoción de aprendizaje significativo; lo que permite que de manera alterna sea el estudiante quien planifique de acuerdo con sus propias demandas y disposición la administración de sus tiempos y la forma de intervención con este recurso. En un tercer apartado se retoma la importancia del proyecto formativo como estrategia didáctica en el desarrollo de competencias, y como una alternativa pertinente y adecuada mediante la cual el estudiante adopta un papel activo al responsabilizarse de su propio proceso de aprendizaje, al concretar la atención y el abordaje de problemas reales.

Finalmente se presenta la planeación de un proyecto formativo en la que confluyen los contenidos de aprendizaje con estrategias innovadoras y con la socialización de los productos alcanzados. Se diseña la propuesta convencidos de que se trata de una estrategia que nos permite el desarrollo y la formación de competencias al enfocarse en la investigación y atención de problemas reales y apoyarse en la tecnología como medio no solo de búsqueda de información, sino de socialización y de aprendizaje colaborativo.

\section{LA MOVILIZACIÓN DE LOS SABERES EN EL DESARROLLO DE LAS COMPETENCIAS}

El enfoque basado en competencias, considera que lo más importante en el proceso del aprendizaje está basado en la forma en que el individuo aplica los conocimientos o saberes en las diferentes áreas de la vida cotidiana y en la solución de problemas que se presentan en el área 
productiva, para Hernández (2019) una de sus características principales es que aborda la relación entre formación y trabajo sin establecer correspondencias rígidas, lo que supone dejar de lado las valoraciones negativas para aquellos que se insertaron en un empleo para el que no se educaron formalmente. Así, más que en el dominio del contenido de los conocimientos por sí mismo; es decir la manera en que el sujeto expresa el dominio de la competencia que ha desarrollado, lo fundamental está en la aplicación práctica de la misma, de esta manera el aprendizaje se convierte en una manera de interactuar con el mundo, pues a medida que aprendemos, se moldean o se modifican nuestras concepciones de los fenómenos.

A partir de esta nueva forma de entender el proceso de aprendizaje se hace necesario dirigir las acciones docentes para lograr la integración del saber desde 3 dimensiones distintas: el saber ser, que implica la dimensión actitudinal, el saber conocer que implica la dimensión conceptual y el saber hacer que implica la dimensión procedimental. De esta manera la meta del docente es cambiar el concepto tradicional de la enseñanza y enfocarse más en reto de promover el aprendizaje en la integración de estas tres dimensiones. La actividad docente estará centrada en el control de estrategias de enseñanza que faciliten el aprendizaje y motiven al alumno a lograr la concientización del mecanismo del saber personal y práctico. López, León y Pérez (2018) demuestran en su estudio la necesidad de impulsar nuevos roles docentes centrados en introducir metodologías activas que favorezcan el papel activo del alumnado, pues la adopción de este enfoque basado en competencias comporta un cambio importante desde el punto de vista pedagógico y didáctico que exige y conlleva a modificar el sentido de la práctica docente.

\section{1 ¿QUE ENTENDEMOS POR EL SABER CONOCER?}

Anteriormente se entendía el saber conocer, como la capacidad de almacenar información que pudiese contener la memoria, como uno de los principales procesos cognoscitivos a activarse para formarse a partir de este saber una concepción que permitiese la interpretación de los fenómenos. El nuevo planteamiento, pretende que se haga una reflexión sobre el sentido que deben tener los conocimientos para lograr los aprendizajes, esto implica que el énfasis está en el procesamiento de la información, más que en el almacenaje de la misma, dicho procesamiento debe incluir la memoria pero integrarla al análisis, la clasificación, la elaboración, reconstrucción y aplicación de la información, es decir, estas operaciones mentales se derivan no solo de la memoria, sino que obliga a la mente a integrar procesos más complejos como la percepción, la atención, la imaginación, la voluntad, la creatividad entre otros. Para Cuevas e Ibarrola (2015) el saber conocer o saberes teóricos se relacionan con elementos cognitivos que pueden ir desde niveles declarativos hasta procesos más complejos de comprensión, análisis y valoración. 
Estos elementos constituyen para Tobón (2010), las herramientas para procesar la información de manera significativa acorde a las expectativas individuales. Se trata así de que el ejercicio docente genere una cultura de promover el saber como un proceso que conlleve además de la adquisición, la transformación y acomodación de la misma; que integre y articule para ello, los diferentes saberes adquiridos en los diversos contextos y situaciones que el estudiante vive. De esta forma la tarea educativa no es en palabras de Zavala y Arnau (2007) “el saber por saber" sino el "saber para hacer". Este tipo de saber utiliza sus propios instrumentos y estrategias, siendo las nociones, las proposiciones, los conceptos y las categorías entre otros, los instrumentos para los cuales se diseñarán estrategias específicas, tales como la selección, la organización, la comparación y la repetición algunas de ellas.

A este respecto es valioso el autocontrol consiente y la regulación personal de procesos como la atención, la memoria y la comprensión como procesos que permiten la generación de aprendizaje significativo.

\section{2 ¿QUE ENTENDEMOS POR EL SABER HACER?}

El saber hacer refiere a una segunda función intelectual que se deriva a partir del saber conocer, adquiere su importancia en la posibilidad de la aplicación del conocimiento, implica por ello la congruencia que se demuestra entre lo que se dice que se sabe y lo que se hace con lo que se sabe. El saber hacer está dirigido a la resolución de problemas de orden tanto teórico como práctico, por lo tanto, las soluciones pueden ser de tipo explicativas y fácticas. Hessen (1977).

En el saber hacer, se buscan metas específicas, se valora el desempeño de la competencia adquirida en la realización de la actividad misma y su pertinencia con la problemática que atiende, ello exige instrumentos basados en los procedimientos y las técnicas. Y las estrategias implican procesos de planeación, regulación y evaluación del proceso de desempeño.

\section{3 ¿QUE ENTENDEMOS POR EL SABER SER?}

El saber ser refiere a la serie de actitudes, posiciones que el sujeto manifiesta de frente a lo que aprende a conocer y a hacer, para que lo hace, a quién beneficia con lo que hace. Se trata de factores internos a la persona, (motivación, identificación) pero también externos, (beneficios, significados) y la combinación entre ambos. Sus principales instrumentos son de carácter afectivo y se basan en los valores, las actitudes y las normas, por ello sus estrategias serán aquellas que vayan dirigidas a potencializar la sensibilización que puede generar la adquisición y el procesamiento de la información, tales como estrategias motivacionales, emocionales y sociales.

Educar en competencias, implica crear experiencias de aprendizaje, para que los estudiantes desarrollen habilidades que les permitan movilizar de forma integral recursos que se consideran 
indispensables para realizar satisfactoriamente las actividades necesarias. Se pretende activar los distintos elementos del aprendizaje, es decir se activan de manera relacionada el saber conocer, el saber hacer y el saber ser. Casanova, Canquiz, Paredes e Inciarte (2018) entienden las competencias como capacidades, habilidades y motivaciones por lo tanto, no basta con saber o saber hacer, es necesario integrar estos saberes reflejadas en actitudes que acompañen las acciones. Esta activación demuestra su integralidad cuando el estudiante además de que comprende como adquirió el dominio del concepto, lo sabe emplear ante las circunstancias externas, y sabe responder con la actitud asertiva que demanda la situación para lograr la meta demandada. La idea es formar en la competencia científica y su aplicabilidad en la realidad, para Sanmarti y Márquez (2017) una persona competente científicamente debe ser capaz de activar estos saberes en una gama de contextos personales, locales y globales y reconocer que la ciencia, la tecnología y la investigación son un elemento esencial de la cultura contemporánea que enmarca gran parte de nuestro pensamiento.

\section{EL USO DE LAS TECNOLOGÍAS Y EL APRENDIZAJE AUTÓNOMO COMO DESEMPEÑO IDÓNEO DEL ESTUDIANTE.}

Hoy en día, las diversas comunidades que integran el complejo tejido social de nuestro planeta se encuentran ante desafíos en donde las transformaciones se rigen a partir de variables que marcan un ritmo acelerado, determinado por el hecho de que actualmente vivimos en lo que se conoce como la era de la información, detonada por el surgimiento de la que es conocida como tercera revolución industrial, donde las TIC juegan un rol protagónico. De acuerdo con Ahumada (2013) la presencia de las TIC ha traído consigo un conjunto de innovaciones promovidas por la globalización, como asimismo por la apuesta de nuevas prácticas de formación desde un enfoque basado en competencias. Por su parte Samekash, (2020) en su estudio demuestra que, a mayor uso de herramientas tecnológicas, mayores logros de aprendizaje autónomo y significativo, de tal manera que el uso de entornos virtuales mejora el rendimiento del aprendizaje.

Con la emergencia de las TIC en esta era de la información surge como consecuencia una nueva sociedad, de acuerdo a UNESCO (2005): la sociedad de la información. La sociedad de la información está basada en las transformaciones y tendencias marcadas por la evolución de la tecnología. Avances como la Internet y la Telefonía móvil tienen como objetivo fundamental en su uso el aplicar técnicas para tratar y transmitir información estableciendo diálogos en donde la interlocución facilite la construcción del conocimiento. Este es quizá el reto más importante que tenemos como sociedad actualmente, el poder transformar sociedades de la información en sociedades del conocimiento en las cuales los individuos tienen la capacidad para identificar, producir, tratar, transformar, difundir y 
utilizar la información con vistas a crear y aplicar los conocimientos necesarios para el desarrollo humano. Esta sociedad, con o nuevas necesidades en la producción y socialización del conocimiento ha traído consigo también nuevos desafíos en el ámbito educativo.

Si bien es cierto que las sociedades de la información y las sociedades del conocimiento abren nuevas posibilidades de desarrollo a partir de los avances tecnológicos y en infraestructura como se menciona en el informe mundial de la UNESCO (2005), también es cierto que estas sociedades deben de garantizar el aprovechamiento compartido del saber, haciendo pleno uso de una libertad de expresión responsable en la que se establecen límites claros de seguridad tanto de los países como de los individuos mismos que los habitan.

Se habla entonces de la gran responsabilidad que deben asumir las instituciones educativas, los docentes, y los estudiantes como agentes protagónicos en la construcción de las sociedades del conocimiento. En este sentido, el enfoque por competencias se ha tomado como instrumento fundamental de una política educativa en la que se replantea la pertinencia de los programas educativos de tal manera que éstos tengan sentido para los docentes y para los estudiantes en la diversidad de los contextos sociales.

El adoptar el enfoque por competencias para supervisar, sistematizar y evaluar el proceso educativo implica que tres actores fundamentales estén en comunión permanente: el estudiante, el docente, y la institución educativa. La institución educativa tiene que gestionar a partir de normas de calidad los recursos y la infraestructura para desarrollar las actividades de aprendizaje en el marco de un diseño curricular compuesto por un proyecto formativo integral que favorezca la movilización del conocimiento, así como de los estudiantes y profesores (Tobón, 2008). El docente por su parte, tiene la obligación de cambiar su rol de transmisor de información para la enseñanza en facilitador para el acceso a la información y orientador en el proceso de construcción del conocimiento. Finalmente, el estudiante debe, a partir de la labor del profesor y de las condiciones que le ofrece la institución educativa, desarrollar una capacidad metacognitiva a través de la cual tenga conciencia de su propio proceso de aprendizaje, lo que le permitirá asumir el protagonismo de su vida constituyéndose como una persona formada integralmente que sabe hacer, sabe conocer, sabe ser y sabe convivir.

A este respecto las TIC propician la posibilidad de este aprendizaje activo, reflexivo además de que promueve actitudes de búsqueda, investigación, comunicación e intercambio y colaboración, de esta manera mediante el uso de las TIC y la plataforma virtual como estrategia didáctica, se generan ambientes colaborativos para el desarrollo de competencias. Así pues, con el diseño de plataformas virtuales se pone al alcance el uso estratégico de herramientas educativas a disposición de los alumnos en la red. 
4 EL PROYECTO FORMATIVO COMO ESTRATEGIA DIDÁCTICA EN EL DESARROLLO DE COMPETENCIAS

La noción de competencia abarca una integración de los dominios en las distintas dimensiones del saber, lo que implica que muestran su idoneidad en la aplicación práctica en la toma de decisiones para la solución de conflictos. Desde esta perspectiva, el método por proyectos representa una forma pertinente y adecuada para expresar el desarrollo de competencias. Mediante la elaboración y el trabajo por proyectos, el estudiante adopta un papel activo pues se hace responsable de su propio aprendizaje, el diseño y construcción de un proyecto formativo permite concretar en problemas o dificultades reales la intervención de lo aprendido en su proceso de formación, convirtiendo este saber en un contenido significativo para quien aprende. Torres, Valbuena, y Rojas (2017) refieren al proyecto formativo como un conjunto articulado de actividades de aprendizaje que se van desplegando en el tiempo para resolver un problema contextualizado y contribuir a desarrollar una o varias competencias. El estudiante experimenta el control y planeación de su proceso de aprendizaje, generando la habilidad de la autonomía y la independencia de este proceso. Se generan formas de aprendizaje académico independientes, es decir sin estar circunscrito al espacio áulico, y al docente como el único sujeto de quien recibe orientación, lo que genera la motivación desde y a partir del sujeto que aprende orientando a la implementación de estrategias propias.

De esta manera, para la realización del trabajo por proyectos, se exige una respuesta que aborde la integración de los saberes para ubicar la posible intervención o solución ante una situación de nuestro contexto. De acuerdo a Tobón (2010) los proyectos formativos permiten la formación y movilización de los distintos saberes, y esto es esencial en el desarrollo de las competencias, pues conllevan a la transversalidad o relación entre las distintas áreas disciplinares, ya que los tópicos del contexto necesariamente abordan contenidos de distintas asignaturas o unidades de aprendizaje, en ello reside la significatividad que los contenidos temáticos pueden generar.

Para el diseño y la factibilidad del trabajo académico basado en proyectos es necesario tener en cuenta que el rol del profesor adquiere características específicas, pues deja de ser el centro del proceso para convertirse en un mediador y facilitador, por lo que debe tener presente, involucrar y concientizar al estudiante en las metas a lograr en cuanto a los aprendizajes esperados y a los objetivos del proyecto; así, el proyecto debe partir del interés propio cuya factibilidad este orientada por el profesor. Por otra parte, es labor del profesor, hacer sentir la necesidad del trabajo colaborativo y la naturaleza socioformativo del trabajo a realizar, así como la intervención y el uso de la tecnología como medio para generar verdaderas condiciones de aprendizaje autónomo y colectivo desarrollando con ello una actitud investigativa y de iniciativa propia. Esta estrategia de trabajo permite que el estudiante logre 
concretar en acciones y productos lo aprendido en teoría, de tal manera que rebasa el aprendizaje mecánico y memorístico al ver metas propuestas convertidas en resultados.

\section{DESCRIPCIÓN DEL PROYECTO FORMATIVO}

En la secuencia didáctica de la unidad de aprendizaje del área de la metodología de la investigación, el trabajo propuesto a través de la estrategia del proyecto formativo, se concibe como la posibilidad de vincular de manera significativa, los contenidos temáticos que describen los elementos metodológicos del proceso investigativo, con la aplicación de estos contenidos en una investigación de una problemática detectada y sentida por el estudiante quien se convierte en un investigador guiado por el docente, en un ambiente de colaboración, al socializar y compartir entre los distintos equipos la diversidad de problemáticas abordadas. Así mismo el programa se complementa en las actividades diseñadas en la plataforma virtual como apoyo didáctico al curso, lo que exige la planeación responsable de la actuación del estudiante en su aprendizaje.

La propuesta exige que el docente diseñe la planeación del proyecto incluyendo una serie de actividades relacionadas con los contenidos, recursos de información necesarios, estrategias colaborativas, organizadas todas en una secuencia didáctica apoyadas en la plataforma virtual, de tal forma que no se convierte en un trabajo complementario extracurricular sino en una guía estratégica pertinente y activa para abordar y concretar los contenidos curriculares. En este sentido, el docente asume dentro y fuera del aula el rol de guía y orientador a través de la retroalimentación constante en este conducto, mientras que el estudiante en interacción, es el actor principal de la construcción del conocimiento.

De esta manera la integración de b-learning como parte de la estrategia de trabajo por proyectos, involucra el uso de la tecnología en los procesos de formación, por lo que esta propuesta incluye garantizar el uso de las redes de computadores, de Internet y de ambientes virtuales de aprendizaje en los procesos directos de formación, porque se propone que aún desde espacios propios fuera de la hora clase, se pueda acceder a los ambientes de trabajo académico, integrar equipos de trabajo, resolver problemas, desarrollar todo tipo de actividades que promuevan la formación de los participantes. De tal forma que el estudiante puede planear su actividad académica utilizando sus propios recursos de tiempo y disposición, generando en ellos una mayor autonomía así, se convierte en el principal administrador de su proceso de aprendizaje bajo la conducción y el apoyo del docente. 


\begin{tabular}{|c|c|c|c|c|c|c|}
\hline \multicolumn{7}{|c|}{ ROYECTO FORMATIVO } \\
\hline \multicolumn{7}{|c|}{ 1. ESTRUCTURA FORMAL } \\
\hline \multicolumn{7}{|c|}{$\begin{array}{l}\text { PERSPECTIVAS INVESTIGATIVAS } \\
\text { Segundo Semestre Psicología Escolarizada }\end{array}$} \\
\hline \multicolumn{4}{|c|}{ Título del proyecto formativo: } & \multicolumn{3}{|c|}{$\begin{array}{c}\text { “JORNADA ESTUDIANTIL: PERSPECTVAS INVESTIGATIVAS EN } \\
\text { PSICOLOGIA" }\end{array}$} \\
\hline \multicolumn{4}{|c|}{ Profesores Responsables del proyecto: } & \multicolumn{3}{|c|}{$\begin{array}{c}\text { RENÉ RODRIGUEZ ZAMORA } \\
\text { LEONOR A. ESPINOZA NÚNEEZ } \\
\end{array}$} \\
\hline \multirow[t]{2}{*}{ Semestre } & \multirow[t]{2}{*}{ Código } & \multirow[t]{2}{*}{ Créditos } & \multirow{2}{*}{\multicolumn{2}{|c|}{ Competencias previas requeridas }} & \multicolumn{2}{|c|}{ Tiempo } \\
\hline & & & & & $\begin{array}{l}\text { Aprendizaje con los } \\
\text { docentes }\end{array}$ & Aprendizaje Autónomo \\
\hline II & & & $\begin{array}{ll}\text { - } & \text { Analiza la pr } \\
\text { integradora } \\
\text { sociales. } \\
\text { - } & \text { Busca y com } \\
\text { - } & \text { Capacidad de } \\
\text { - } & \text { Utiliza recurs } \\
& \text { tareas. } \\
\end{array}$ & $\begin{array}{l}\text { oblemática psicológica desde una perspectiva } \\
\text { de los procesos psicológicos, biológicos y } \\
\text { prende información de fuentes diversas. } \\
\text { organización y planificación. } \\
\text { sos que ofrecen las TIC para la realización de }\end{array}$ & & \\
\hline \multicolumn{7}{|c|}{ Naturaleza del proyecto formativo: } \\
\hline \multicolumn{7}{|c|}{ Es un proyecto global ya que aborda competencias específicas y genéricas, con énfasis en lo interdisciplinario, aplicativo, en un contexto comunitario. } \\
\hline \multicolumn{7}{|c|}{ 2. COMPETENCIAS QUE PRETENDE FORMAR: } \\
\hline \multicolumn{4}{|c|}{$\begin{array}{l}\text { Competencia genérica de énfasis: } \\
\text { 1. Conoce y analiza críticamente las características de los } \\
\text { métodos de investigación en el estudio de lo psicológico. }\end{array}$} & \\
\hline \multicolumn{4}{|c|}{$\begin{array}{l}\text { 2. Manifiesta habilidades y actitudes favorables para el } \\
\text { aprendizaje y el trabajo colaborativo e interdisciplinar. }\end{array}$} & \multicolumn{3}{|c|}{$\begin{array}{l}\text { Se consideran los siguientes criterios: } \\
\text { - Investiga diversas problemáticas psicosociales poniendo en práctica métodos y técnicas de } \\
\text { investigación cualitativa y cuantitativa. } \\
\text { - Analiza la problemática psicológica desde una perspectiva integradora de los procesos psicológicos, } \\
\text { biológicos y sociales. } \\
\text { - Diseña y desarrolla investigaciones básicas y aplicadas en las distintas áreas de la psicología: } \\
\text { clínica, educativa, comunitaria, social y organizacional. } \\
\text { - Aplicar con efectividad, rigor metodológico y principios éticos las principales técnicas y } \\
\text { - Drocedimientos establecidos para la detección de la problemática psicosocial. } \\
\text { - Trunde los resultados de la investigación realizada a través de medios formales e informales. } \\
\text { - Trabaja de manera colaborativa con otras personas al compartir un propósito común. }\end{array}$} \\
\hline \multicolumn{7}{|c|}{ Nivel de dominio esperado: } \\
\hline \multicolumn{7}{|c|}{ Se pretende que los estudiantes construyan la competencia de énfasis del proyecto formativo por lo menos hasta un grado Resolutivo } \\
\hline
\end{tabular}


- Aspectos clave del proyecto:

Pretende identificar los pasos metodológicos para la realización de una investigación y su utilidad para conocer especificidades de una problemática psicosocial Además de establecer un proyecto de participación en la socialización de los resultados en la búsqueda de alternativas de solución, mediante la organización de jornadas donde exponga y comparta sus productos.

- Problema que pretende abordar:

La mayor parte de los contenidos analizados en las distintas unidades de aprendizaje llegan a resultados que no se vinculan con situaciones reales y de interés de los sujetos, los resultados y alcances de los ejercicios de investigación relacionados con el análisis de alguna problemática no son socializados entre la comunidad escolar.

\section{ACTIVIDADES DEL PROYECTO:}

FASE 1: Direccionamiento:

\begin{tabular}{|c|c|c|c|c|c|c|}
\hline Actividades de Aprendizaje con el Docente & $\begin{array}{l}\text { Actividades de aprendizaje } \\
\text { autónomo e independiente }\end{array}$ & 5. Estruc & ura de la Evalu & ción & & \\
\hline \multirow{4}{*}{ 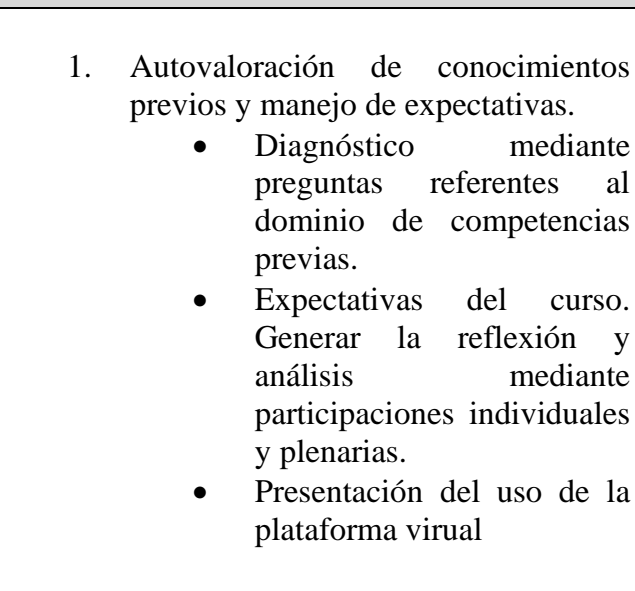 } & \multirow{4}{*}{$\begin{array}{l}\text { 1. Recuperación de los } \\
\text { conocimientos previos de } \\
\text { acuerdo a los resultados de } \\
\text { su autovaloración para el } \\
\text { buen desempeño del curso. } \\
\text { 2. Presentación de un escrito } \\
\text { donder establece } \\
\text { compromiso de } \\
\text { participación acuerdo a las } \\
\text { expectativas del proyecto. } \\
\text { 3. Exploración y contacto } \\
\text { con el diseño de la } \\
\text { plataforma virtual }\end{array}$} & \multicolumn{5}{|c|}{$\begin{array}{l}\text { Criterio: } \\
\qquad \quad \text { Trabaja de manera colaborativa con otras personas al compartir un propósito } \\
\quad \text { común. } \\
\text { Evidencia } \\
\text { Documento de autovaloración }\end{array}$} \\
\hline & & Pre-formal & Receptivo & Resolutivo & Autónomo & Estratégico \\
\hline & & \begin{tabular}{lr}
\multicolumn{2}{l}{ El estudiante se } \\
limita \\
escuchar sin \\
participar.
\end{tabular} & $\begin{array}{l}\text { El estudiante } \\
\text { reconoce solo } \\
\text { parte de su } \\
\text { nivel de } \\
\text { aprendizaje } \\
\text { sin sentir la } \\
\text { necesidad de } \\
\text { mejorar }\end{array}$ & $\begin{array}{l}\text { El estudiante } \\
\text { reconoce su } \\
\text { nivel de } \\
\text { aprendizaje } \\
\text { sin } \\
\text { estrategias } \\
\text { claras a } \\
\text { seguir. }\end{array}$ & $\begin{array}{l}\text { El estudiante } \\
\text { reconoce su } \\
\text { nivel de } \\
\text { aprendizaje y } \\
\text { establece } \\
\text { compromisos } \\
\text { para mejorar. }\end{array}$ & $\begin{array}{l}\text { El estudiante } \\
\text { reconoce su nivel } \\
\text { de aprendizaje, } \\
\text { estableciendo } \\
\text { compromisos con } \\
\text { una actitud asertiva } \\
\text { hacia las críticas. }\end{array}$ \\
\hline & & $\%$ & $\%$ & $\%$ & $\%$ & $\%$ \\
\hline $\begin{array}{l}\text { 2. Socialización y comprensión de la } \\
\text { competencia a desarrollar en el curso, } \\
\text { proponiendo el desarrollo del } \\
\text { proyecto y la toma de acuerdos para el } \\
\text { objetivo y las actividades a realizar. }\end{array}$ & $\begin{array}{l}\text { 4. } \begin{array}{l}\text { Discute y analiza los } \\
\text { conceptos básicos del } \\
\text { proceso investigativo. }\end{array} \\
\text { 5. } \begin{array}{l}\text { Elaborar el diseño del } \\
\text { anteproyecto a llevar a }\end{array} \\
\text { cabo durante el semestre, } \\
\text { precisando la problemática }\end{array}$ & \multicolumn{5}{|c|}{$\begin{array}{l}\text { Criterio } \\
\quad \text { Analiza la problemática psicológica desde una perspectiva integradora de los } \\
\text { procesos psicológicos, biológicos y sociales. } \\
\text { Evidencia } \\
\text { Presentación del diseño de anteproyecto a realizar conteniendo las actividades principales } \\
\text { y las normas a cumplir. }\end{array}$} \\
\hline
\end{tabular}


LATIN AMERICAN

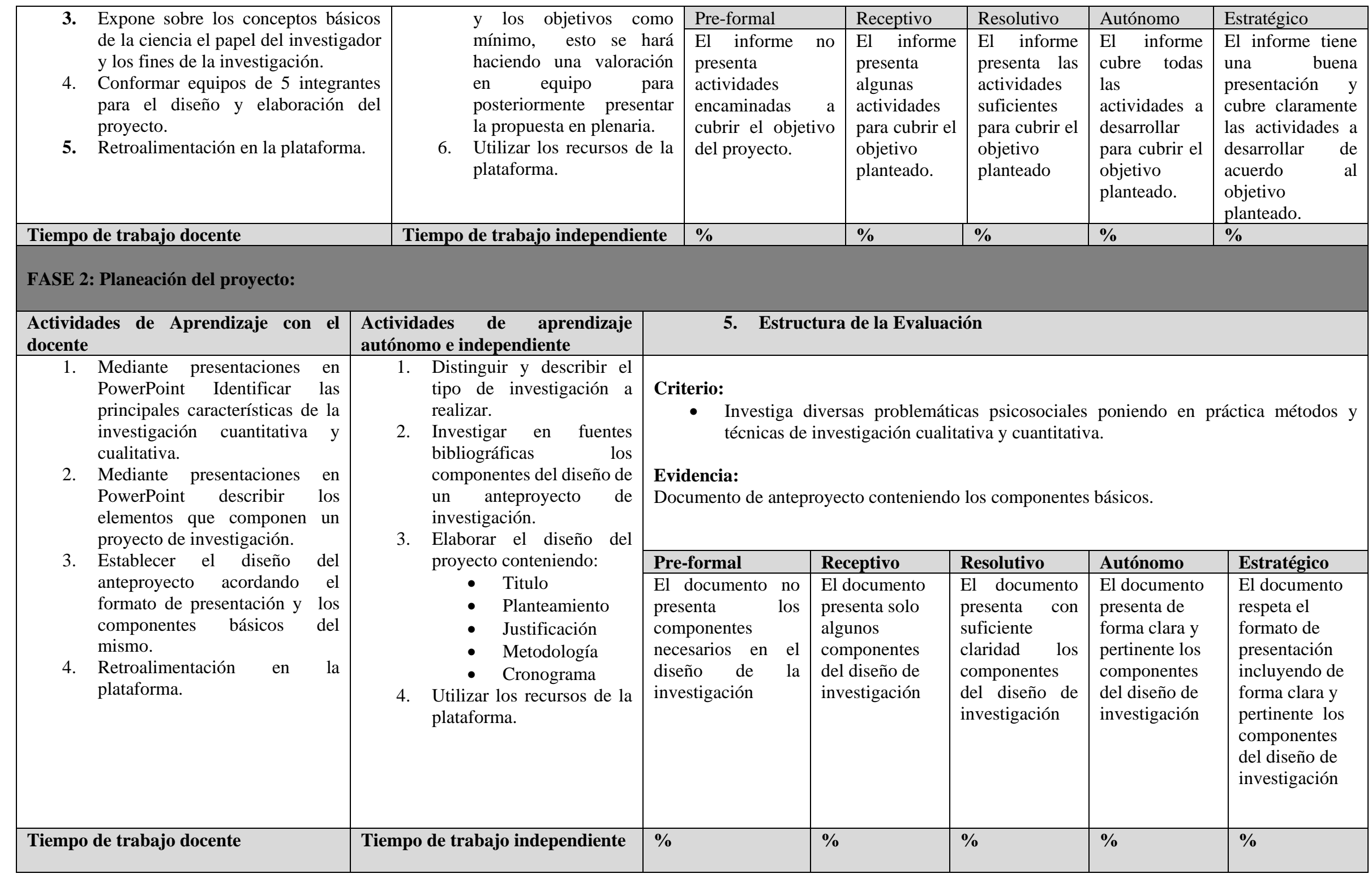




\begin{tabular}{|c|c|c|c|c|c|c|c|c|}
\hline \multicolumn{2}{|c|}{ Actividades de aprendizaje con el docente } & \multicolumn{2}{|l|}{$\begin{array}{l}\text { Actividades de aprendizaje } \\
\text { autónomo e independiente }\end{array}$} & \multicolumn{5}{|c|}{$5 \quad$ Estructura de la Evaluación } \\
\hline \multirow{3}{*}{\multicolumn{2}{|c|}{$\begin{array}{l}\text { 1. Identificar los componentes esenciales de } \\
\text { un proyecto mediante la presentación de } \\
\text { diagramas o mapas mentales. } \\
\text { 2. Guiar y orientar a los equipos en el } \\
\text { proceso metodológico. } \\
\text { 3. Identificar los principales criterios de } \\
\text { escritura para la elaboración de un } \\
\text { informe de investigación con rigurosidad } \\
\text { científica. } \\
\text { 4. Retroalimentación en la plataforma. }\end{array}$}} & \multirow{3}{*}{\multicolumn{2}{|c|}{$\begin{aligned} & \text { 1. } \text { Elaboración del marco } \\
& \text { teórico. } \\
& \text { 2. } \text { Definición del diseño de } \\
& \text { investigación. } \\
& \text { 3. }\end{aligned}$}} & \multicolumn{5}{|c|}{$\begin{array}{l}\text { Criterio: } \\
\text { - Diseña y desarrolla investigaciones básicas y aplicadas en las distintas áreas } \\
\text { de la psicología: clínica, educativa, comunitaria, social y organizacional. } \\
\text { - Aplica con efectividad, rigor metodológico y principios éticos las } \\
\text { principales técnicas y procedimientos establecidos para la detección de la } \\
\text { problemática psicosocial. } \\
\text { - Redacta conclusiones de la investigación realizada. } \\
\text { Evidencia: informe de investigación }\end{array}$} \\
\hline & & & & Pre-formal & Receptivo & Resolutivo & Autónomo & \begin{tabular}{|l} 
Estratégico \\
\end{tabular} \\
\hline & & & & $\begin{array}{l}\text { El texto } \\
\text { presenta } \\
\text { una } \\
\text { redacción } \\
\text { inconsistent } \\
\text { e y con } \\
\text { errores en la } \\
\text { mayor parte } \\
\text { de sus } \\
\text { elementos }\end{array}$ & $\begin{array}{l}\text { El texto es lo } \\
\text { suficienteme } \\
\text { nte claro } \\
\text { manejando } \\
\text { errores en } \\
\text { cada } \\
\text { elemento. }\end{array}$ & $\begin{array}{l}\text { El texto es } \\
\text { coherente en } \\
\text { al menos } 3 \\
\text { de sus } \\
\text { elementos }\end{array}$ & $\begin{array}{l}\text { El texto } \\
\text { contempla la } \\
\text { mayor parte } \\
\text { de los } \\
\text { elementos con } \\
\text { la claridad } \\
\text { suficiente y } \\
\text { con no más de } \\
5 \text { errores } \\
\text { ortográficos }\end{array}$ & $\begin{array}{l}\text { el texto presenta } \\
\text { todos los } \\
\text { elementos con } \\
\text { claridad en las } \\
\text { ideas, sin errores } \\
\text { ortográficos y de } \\
\text { estilo. }\end{array}$ \\
\hline \multicolumn{2}{|l|}{ Tiempo de trabajo docente } & \multicolumn{2}{|c|}{ Tiempo de trabajo independiente } & $\%$ & $\%$ & $\%$ & $\%$ & $\%$ \\
\hline \multicolumn{9}{|l|}{ FASE 4 : Socialización del proyecto } \\
\hline $\begin{array}{l}\text { Actividades de aprendizaje con } \\
\text { el docente }\end{array}$ & \multicolumn{2}{|c|}{$\begin{array}{l}\text { Actividades de aprendizaje } \\
\text { autónomo e independiente }\end{array}$} & \multicolumn{6}{|c|}{ Estructura de la Evaluación } \\
\hline \multirow[t]{2}{*}{$\begin{array}{l}\text { 1. Organiza a los estudiantes } \\
\text { para el diseño y la } \\
\text { logística del evento "1er } \\
\text { Jornada Estudiantil, } \\
\text { perspectivas investigativas } \\
\text { " } \\
\text { 2. Promueve la estructura de } \\
\text { un comité organizador }\end{array}$} & \multirow{2}{*}{\multicolumn{2}{|c|}{$\begin{array}{l}\text { 1. Colaboración para el diseño y la } \\
\text { operatividad del evento 1er } \\
\text { Jornada estudiantil, perspectivas } \\
\text { investigativas" } \\
\text { 2. Nombramiento } \\
\text { involucramiento del comité } \\
\text { organizador. } \\
\text { 3. Diseño de la logística. }\end{array}$}} & \multicolumn{6}{|c|}{$\begin{array}{l}\text { Criterio: } \\
\text { - Trabaja de manera colaborativa con otras personas al compartir un propósito } \\
\text { común. } \\
\text { - Difunde los resultados de la investigación realizada a través de medios formales e } \\
\text { informales } \\
\text { Evidencia: } \\
\text { Organización del evento “Jornadas Estudiantiles" }\end{array}$} \\
\hline & & & Pre-for & & & & Autónomo & Estratégico \\
\hline
\end{tabular}




\begin{tabular}{|c|c|c|c|c|c|c|}
\hline & $\begin{array}{l}\text { 4. Participación en la exposición de } \\
\text { su trabajo. } \\
\text { 5. Participación en el debate. }\end{array}$ & $\begin{array}{l}\text { El estudiante } \\
\text { se limita a } \\
\text { escuchar sin } \\
\text { participar. }\end{array}$ & $\begin{array}{l}\text { El estudiante } \\
\text { reconoce parte de } \\
\text { su nivel de } \\
\text { aprendizaje sin } \\
\text { sentir la necesidad } \\
\text { de comprometerse }\end{array}$ & $\begin{array}{l}\text { El estudiante } \\
\text { reconoce su } \\
\text { nivel de } \\
\text { aprendizaje } \\
\text { sin estrategias } \\
\text { claras a seguir. }\end{array}$ & $\begin{array}{l}\text { El estudiante } \\
\text { reconoce su } \\
\text { nivel de } \\
\text { aprendizaje y } \\
\text { establece } \\
\text { compromisos } \\
\text { de } \\
\text { participación. }\end{array}$ & $\begin{array}{l}\text { El estudiante } \\
\text { reconoce su nivel } \\
\text { de aprendizaje, } \\
\text { estableciendo } \\
\text { compromisos con } \\
\text { una actitud } \\
\text { asertiva hacia las } \\
\text { críticas. }\end{array}$ \\
\hline Tiempo de trabajo docente & Tiempo de trabajo independiente & $\%$ & $\%$ & $\%$ & $\%$ & $\%$ \\
\hline
\end{tabular}

\section{RECURSOS}

- Talento humano requerido

- Equipo tecnológico

- Presupuesto

\section{ACUERDO DE NORMAS}

- Asistencia obligatoria las sesiones de explicación metodológica y presentaciones de avances.

- Utilización de los recursos y las actividades diseñadas en apoyo al curso en plataforma virtual.

- Asistencia y participación en la organización del evento final.

- Participación en la presentación de avances y en la exposición final.

- Respetar las fechas de entrega establecidas para cada una de las etapas del proyecto. 


\section{EL USO DE B-LEARNING PARA FORTALECER EL PROYECTO FORMATIVO}

Es evidente que en estas nuevas formas de enseñanza colaborativa, en el marco de la era de la información, las tecnologías representan una posibilidad de acercamiento directo y permanente a los actores involucrados en la producción del conocimiento, mediante ellas el estudiante amplía la gama de opciones para aprender mediante la interacción. Santoveña (2012), relaciona en su estudio la metodología didáctica utilizada con apoyo en curso virtual y el rendimiento académico, concluyendo que el aula virtual facilita el acceso a la información y la aclaración de dudas, mediante la interacción. Así en el paradigma constructivista, el proceso de aprendizaje escolar está enfocado en el carácter activo del sujeto que aprende, de tal forma que este proceso es producto de acciones compartidas entre docente y estudiante así como entre el estudiante y sus pares. Desde este marco, el docente tendrá como principal responsabilidad generar las condiciones para que se establezca el ambiente de aprendizajes, donde la tecnología mediante herramientas específicas como el b-learning, conforman una estrategia didáctica cuyo componente principal es la interacción en línea como una forma de respaldar el aprendizaje presencial y de generar con ello el aprendizaje autónomo o independiente. En este sentido en un curso b-learning el profesor deja de ocupar el papel central, dejando ese espacio a los estudiantes, se provoca con ello que los alumnos adquieran mayor responsabilidad para conducir el desarrollo del curso hacia el éxito.

Con respecto al proyecto formativo, en la idea de generar condiciones para el aprendizaje autónomo, colaborativo y orientado en la investigación, se diseña como parte de la estrategia didáctica la plataforma para aula virtual, en ella se pone al acceso del estudiante los recursos indispensables para su formación en el área de la investigación, así mismo, una secuencia didáctica que permite la realización de actividades en apoyo al curso presencial. En ella el estudiante se convierte en el administrador de sus propios tiempos y su dinámica de aprendizaje, por otra parte, representa como ventaja la posibilidad de continuar activo en el curso aún cuando por determinadas circunstancias no se pudo garantizar la presencia en aula física, por algunos días. De esta forma, se genera en el estudiante la interacción presencial apoyada en la interacción virtual, como principales componentes del proceso cognitivo, incrementando la motivación y una actitud positiva hacia la planificación de su proceso de aprender.

\section{CONCLUSIONES}

- Educar en competencias, implica crear experiencias de aprendizaje, para que los estudiantes desarrollen habilidades que les permitan movilizar, de forma integral recursos que se consideran indispensables para realizar satisfactoriamente las actividades necesarias, activando las tres dimensiones del saber. 
- El uso de las TIC propicia la posibilidad del aprendizaje activo, reflexivo además de que promueve actitudes de búsqueda, investigación, comunicación e intercambio y colaboración, de esta manera mediante el uso de las TIC y la plataforma virtual como estrategia de apoyo didáctico, se generan ambientes colaborativos para el desarrollo de competencias.

- El método por proyectos representa una forma pertinente y adecuada para expresar el desarrollo de competencias. El diseño y construcción de un proyecto formativo permite concretar en problemas o dificultades reales la intervención de lo aprendido en su proceso de formación. Se generan formas de aprendizaje académico independientes, es decir sin estar circunscrito al espacio áulico, y al docente como el único sujeto de quien recibe orientación, lo que genera la motivación desde y a partir del sujeto que aprende orientando a la implementación de estrategias propias.

- La propuesta exige que el docente diseñe la planeación del proyecto incluyendo una serie de actividades relacionadas con los contenidos, recursos de información necesarios, estrategias colaborativas, organizadas todas en una secuencia didáctica apoyadas en la plataforma virtual, de tal forma que no se convierte en un trabajo complementario extracurricular sino en una guía estratégica pertinente y activa para abordar y concretar los contenidos curriculares, que exige además un cambio en el rol tradicional del maestro. 


\section{REFERENCIAS}

- $\quad$ Ahumada Torres, M. E. (2013). Las TIC en la formación basada en competencias. Revista de la Universidad de La Salle, 2013(60), 141-157.

- Casanova, I. I., Canquiz, L., Paredes Chacín, Í., \& Inciarte González, A. (2018). Visión general del enfoque por competencias en Latinoamérica.

- Cuevas de la Garza, J. F., \& de Ibarrola Nicolín, M. (2015). Aprender en la simultaneidad: la perspectiva de los estudiantes que trabajan sobre los saberes y competencias que construyen. Revista mexicana de investigación educativa, 20(67), 1157-1186.

- Hernández Martínez, D. C. (2019). Del enfoque adecuacionista al enfoque basado en competencias: una mirada a la inserción al mercado laboral de los egresados de Sociología. Sociológica (México), 34(98), 189-219.

- Hessen Johannes. 1977. Teoría del Conocimiento. Editores Unidos, S.A., México, primera edición. Informe mundial de la UNESCO. (2005). Hacia las sociedades del conocimiento. Francia: UNESCO, pp. 17-47.

- $\quad$ López López, M. del C., León Guerrero, M. J., \& Pérez García, M. P. (2018). El enfoque por competencias en el contexto universitario español. La visión del profesorado. Revista de Investigación Educativa, 36(2), 529-545. https://doi.org/10.6018/rie.36.2.314351

- Sanmarti Puig, N., \& Márquez Bargalló, C. (2017). Aprendizaje de las ciencias basado en proyectos: del contexto a la acción. Apice, 1(1), 3-16.

- Santoveña Casal Sonia Ma. 2012 Repercusión de la metodología didáctica con apoyo de cursos virtuales de aprendizaje en el rendimiento de los estudiantes. Revista de Currículum y Formación del Profesorado. Vol. 16, No 3

- $\quad$ Samekash, M. L. W. (2020). Uso de whatsapp para mejorar el aprendizaje autónomo en los jóvenes universitarios. EDUCARE ET COMUNICARE: Revista de investigación de la Facultad de Humanidades, 8(1), 78-87.

- Tobón Tobón Sergio 2010 "Movilización de los saberes y práctica metacognitiva” en Formación Integral y Competencias Colombia. Eco Ediciones.

- Tobón Tobón, Sergio. (2008). La forma basada en competencias en la educación superior. El enfoque complejo. México: Universidad de Guadalajara, pp. 1-30.

- $\quad$ Torres, S. A. C., Valbuena, S. J., \& Rojas, L. F. C. (2017). La metodología de proyectos formativos como estrategia didáctica para la formación y evaluación de competencias: un caso de aplicación. Encuentro Internacional de Educación en Ingeniería.

Zabala Antoni y Arnau Laia. (2007). 11 Ideas Clave, Cómo aprender y enseñar competencias. Barcelona: Graó de IRIF, S.L. 